\title{
DILATOMETRIC INVESTIGATIONS OF OVERCRITICAL BEHAVIOUR IN $\left[\mathrm{N}\left(\mathrm{C}_{2} \mathrm{H}_{5}\right)_{4}\right]_{2} \mathrm{CuCl}_{4}$ CRYSTALS
}

\author{
R. Poprawski ${ }^{a}$, A. LIBER ${ }^{b}$ AND E. MAeEK ${ }^{a}$ \\ anstitute of Physics, Wrocław University of Technology \\ Wybrzeze Wyspiańskiego 27, 50-370 Wrocław, Poland \\ ${ }^{b}$ Computer Science Department, Wrocław University of Technology \\ Wybrzeże Wyspiańskiego 27, 50-370 Wrocław, Poland
}

(Received February 23, 2000)

The results of experimental investigation of thermal expansion of $\left[\mathrm{N}\left(\mathrm{C}_{2} \mathrm{H}_{5}\right)_{4}\right]_{2} \mathrm{CuCl}_{4}$ crystals in a wide temperature range are presented. The anomaly of thermal deformation typical of first order phase transition at $258 \mathrm{~K}$ and additional continuous anomaly at $195 \mathrm{~K}$ were observed. It was shown that low temperature anomalies of physical properties of $\left[\mathrm{N}\left(\mathrm{C}_{2} \mathrm{H}_{5}\right)_{4}\right]_{2} \mathrm{CuCl}_{4}$ crystals can be described as overcritical trace of isomorphous phase transitions. Results of numerical analysis of phenomenological model of improper ferroelastic exhibiting isomorphous phase transitions are presented and compared with experimentally determined temperature dependencies of spontaneous deformation of $\left[\mathrm{N}\left(\mathrm{C}_{2} \mathrm{H}_{5}\right)_{4}\right]_{2} \mathrm{CuCl}_{4}$ crystals.

PACS numbers: $61.68 .+\mathrm{n}$

\section{Introduction}

Most publications on solid state phase transitions unquestionably concern transitions with a symmetry change, called structural transitions. Phase transitions without any symmetry change, called isomorphic or isostructural transitions, are unique in the solid state.

Isostructural phase transition in solid state for the first time was observed in metallic cerium [1]. Cerium phase diagram was determined in the pressuretemperature plane and a critical point similar to that for the liquid-gas system was observed. Based on calorimetric studies Tatsumi et al. [2] showed that there was a critical point in $\mathrm{SnCl}_{2} \cdot\left(\mathrm{H}_{2} \mathrm{O}\right)_{x}\left(\mathrm{D}_{2} \mathrm{O}\right)_{2-x}$ crystals, provided that the role of pressure in the liquid-gas system was played by deuterium concentration. Isostructural phase transition and a critical point were also observed in $\mathrm{Ca}_{2} \mathrm{~Pb}\left(\mathrm{C}_{2} \mathrm{H}_{5} \mathrm{COO}\right)_{6}$ crystals [3]. 
An overcritical trace of the isostructural phase transition was observed in $\left(\mathrm{CH}_{3} \mathrm{NH}_{3}\right)_{5} \mathrm{Bi}_{2} \mathrm{Cl}_{11}$ (PMACB) $[4,5]$ and $\left(\mathrm{CH}_{3} \mathrm{NH}_{3}\right)_{5} \mathrm{Bi}_{2} \mathrm{Br}_{11}$ (PMABB) crystals [6] - proper ferroelectrics. Recently, we have shown that the PMACB crystals [7] can be carried through a critical point due to irradiation.

In $\left[\mathrm{N}\left(\mathrm{C}_{2} \mathrm{H}_{5}\right)_{4}\right]_{2} \mathrm{CuCl}_{4}$ crystals labelled as (TEA) ${ }_{2} \mathrm{CuCl}_{4}$ anomaly of specific heat characteristic of first-order phase transition of order-disorder type at $258 \mathrm{~K}$ and additional anomaly of specific heat with maximum at $196 \mathrm{~K}$ were observed by differential thermal analysis (DTA) and adiabatic calorimetry by Melia and Merrifield [8,9]. Not long ago Mahoui et al. published works on structure and physical properties of (TEA) ${ }_{2} \mathrm{CuCl}_{4}$ crystals [10-12].

In the high-temperature phase the crystal belongs to a $P 4_{2} / n m c(Z=2)$ space group of tetragonal system, whereas at temperatures below $258 \mathrm{~K}$ the crystal belongs to a Pnna $(Z=4)$ space group of rhombic system [11] or to $P 4 / n(Z=4)$ space group of tetragonal system [13]. In the phase below $258 \mathrm{~K}(\mathrm{TEA})_{2} \mathrm{CuCl}_{4}$ crystals belongs to improper ferroelastics [9] or ferroelastoelectrics [14].

Around $203 \mathrm{~K}$ specific heat [9, 11], thermal expansion [15], and dielectric anomaly [16] in (TEA) ${ }_{2} \mathrm{CuCl}_{4}$ crystal were observed, and we treated the anomalies as the sign of the isostructural transition $[11,12]$. Based on the structural studies $[11,14]$ it was shown that the crystal symmetry below and above $203 \mathrm{~K}$ remained the same.

It is worth noting that (TEA) ${ }_{2} \mathrm{CuCl}_{4}$ crystal specific heat and thermal expansion coefficient around $203 \mathrm{~K}$ remain continuous, so it cannot be stated that the phase transition is of the first order. Since isostructural transitions have to be of the first order, the observed phenomenon is probably an "overcritical trace" of isostructural phase transition. Looking at the specific heat and thermal expansion coefficient anomalies it can be concluded that the crystal is very close to the critical point.

Mahoui et al. [12] suggest an expansion of free energy around two-component order parameter considering the fourth-power component to describe physical properties of (TEA) ${ }_{2} \mathrm{CuCl}_{4}$ crystal. The interaction of the order parameter with external stress was taken into consideration. From minimum condition of free energy expansion they obtained the following solutions:

$$
\begin{aligned}
& \rho_{1}=0, \quad u_{6}=0, \\
& \cos (4 \varphi)=-1, \quad \rho_{1}^{2}=-\frac{\alpha}{2\left(\beta-\beta^{\prime}\right)}, \quad u_{6}= \pm \alpha \rho_{1}^{2} .
\end{aligned}
$$

The solution (1a) describes paraelastic phase, solutions (1b) describe improper ferroelastic one. In the solutions (1a) and (1b) $a, \beta$ and $\beta^{\prime}$ are constants (the expansion coefficients), $\rho_{1}$ and $\varphi$ are the module and the phase of the order parameter respectively, whereas $u_{6}$ denotes component of the spontaneous deformation tensor.

Considering solution (1b), the square order parameter module $\rho_{1}^{2}$ is linearly connected with the spontaneous shear deformation at $x y$ plane $u_{6}=\frac{1}{2} u_{12}$.

Temperature dependence of this deformation can be determined in dilatometric studies. As mentioned above, (TEA) ${ }_{2} \mathrm{CuCl}_{4}$ crystal dilatometric measurements were carried out by Tylczyński and Biskupski [15], however they measured 
temperature dependence of the thermal deformation along the [100] and [111] directions, whereas the deformation connected with the order parameter is the one along the [110] direction.

According to the phase transition theory $[17,18]$ the free energy expansion used in [12] can be employed only for description of the second-order phase transition. As it was mentioned above, calorimetric [9], dilatometric [15], and dielectric measurements [16] proved that the phase transition in (TEA) ${ }_{2} \mathrm{CuCl}_{4}$ crystals at $258 \mathrm{~K}$ was of the first order. For description of the structural phase transition of the first order and low-temperature anomalies in physical properties of (TEA) ${ }_{2} \mathrm{CuCl}_{4}$ crystals, components with the order parameter in the tenth power should be considered in the free energy expansion.

The aim of this work is to analyse the free energy expansion which can describe a structural phase transition of the first order and an overcritical trace of isostructural phase transition for improper ferroelastics, as well as can determine (TEA $)_{2} \mathrm{CuCl}_{4}$ crystal thermal deformation and compare experimental results with theoretical data.

It should be pointed out that (TEA) ${ }_{2} \mathrm{CuCl}_{4}$ crystals are the first improper ferroelastics or ferroelastoelectric exhibiting overcritical phenomena.

\section{Experimental and measurement results}

The initial material for growing the crystals was obtained from chemical reaction of ethylammonium chloride $\left(\left(\mathrm{C}_{2} \mathrm{H}_{5}\right)_{4} \mathrm{~N}\right) \mathrm{Cl}$ with cupric chloride $\mathrm{CuCl}_{2}$. Anhydrous ethanol was used as a solvent.

The (TEA) ${ }_{2} \mathrm{CuCl}_{4}$ single crystals were grown from the anhydrous ethanol solution by isothermal vaporization at about $300 \mathrm{~K}$. The process gave yellow, optically uniform, and transparent single crystals of $1-2 \mathrm{~cm}$ in size.

The crystals were oriented with a conoscope technique. Samples of the length from 4.3 to $4.75 \mathrm{~mm}$, cut perpendicularly to the [001] and [110] directions were chosen for dilatometric studies. Measurements were carried out with a computer-controlled capacitance dilatometer. Details are described in [19]. The samples were placed in a measurement chamber which was evacuated and filled with dry gaseous helium. The measurements were performed within temperature range of 130 to $290 \mathrm{~K}$.

Figure 1 presents the results of thermal deformation measurements for (TEA) ${ }_{2} \mathrm{CuCl}_{4}$ crystal along the [001] direction in the temperature range from 130 to $280 \mathrm{~K}$. A great jump in deformation is observed at the structural phase transition at temperature $258 \mathrm{~K}$ and a continuous anomaly in thermal deformation can be distinguished around $196 \mathrm{~K}$.

Figure 2 shows temperature dependence of the spontaneous deformation for (TEA) ${ }_{2} \mathrm{CuCl}_{4}$ crystal along the [001] direction, i.e., the difference between the crystal thermal deformation approximated linearly from the paraelastic phase and the experimental curve. At $258 \mathrm{~K}$ a jump-like relative change of crystal size in [001] direction occurred, reaching the value of 0.0038 . Figure 3 illustrates the temperature dependence of (TEA $)_{2} \mathrm{CuCl}_{4}$ crystal thermal expansion coefficient in [001] direction. 


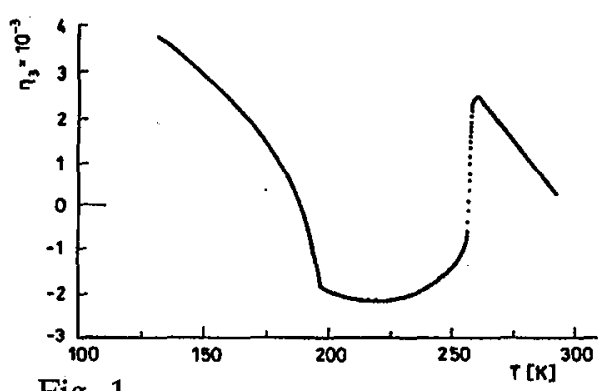

Fig. 1

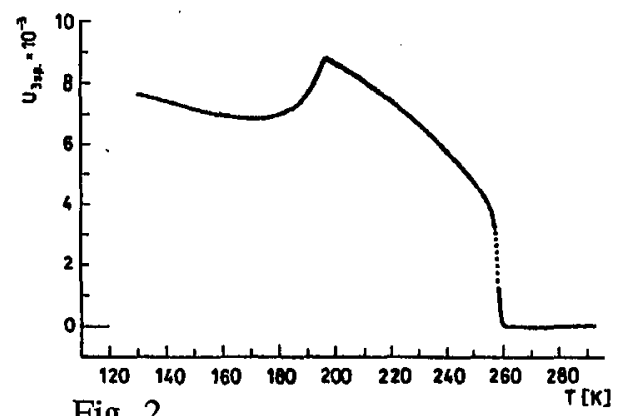

Fig. 2

Fig. 1. Temperature dependence of (TEA) ${ }_{2} \mathrm{CuCl}_{4}$ crystal thermal deformation along the [001] direction.

Fig. 2. Temperature dependence of (TEA) ${ }_{2} \mathrm{CuCl}_{4}$ crystal spontaneous deformation approximated linearly from paraelastic phase along the [001] direction.

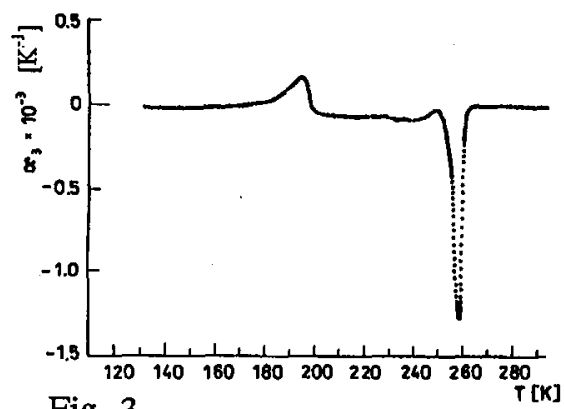

Fig. 3

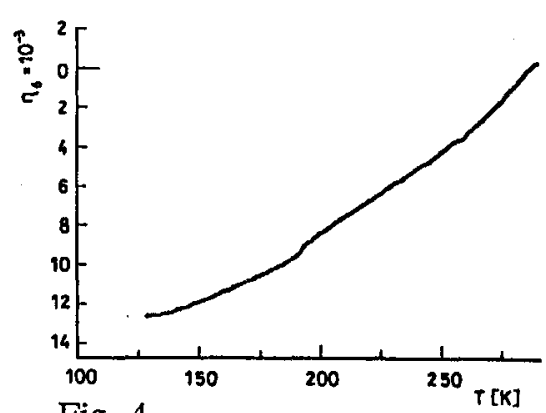

Fig. 4

Fig. 3. (TEA) ${ }_{2} \mathrm{CuCl}_{4}$ crystal thermal expansion coefficient along the [001] direction as a function of temperature.

Fig. 4. Temperature dependence of $(\mathrm{TEA})_{2} \mathrm{CuCl}_{4}$ crystal thermal deformation along the $[110]$ direction.

Results of thermal deformation measurement along the [110] direction for (TEA) ${ }_{2} \mathrm{CuCl}_{4}$ crystal within temperature range from 130 to $280 \mathrm{~K}$, are shown in Fig. 4. There are two small anomalies, one around the ferroelastic phase transition at $258 \mathrm{~K}$, the other at about $196 \mathrm{~K}$. Figure 5 a presents temperature dependence of $(\mathrm{TEA})_{2} \mathrm{CuCl}_{4}$ crystal spontaneous deformation along the [110] direction in structural phase transition region approximated linearly from the paraelastic phase. The jump of relative size of the crystal in [110] direction reaching the value of 0.00035 is one order smaller than that observed in [001] direction. Temperature dependence of spontaneous deformation in [110] direction in the vicinity of the low-temperature anomaly is presented in Fig. 5b. Figure 6 illustrates the temperature dependence of (TEA) ${ }_{2} \mathrm{CuCl}_{4}$ crystal thermal expansion coefficient in [110] direction.

Based on the results presented above, i.e., the temperature dependencies of the spontaneous deformation and thermal expansion coefficient, we confirm the existence of the structural phase transition of the first order at $T_{\mathrm{p}}=258 \mathrm{~K}$ and 

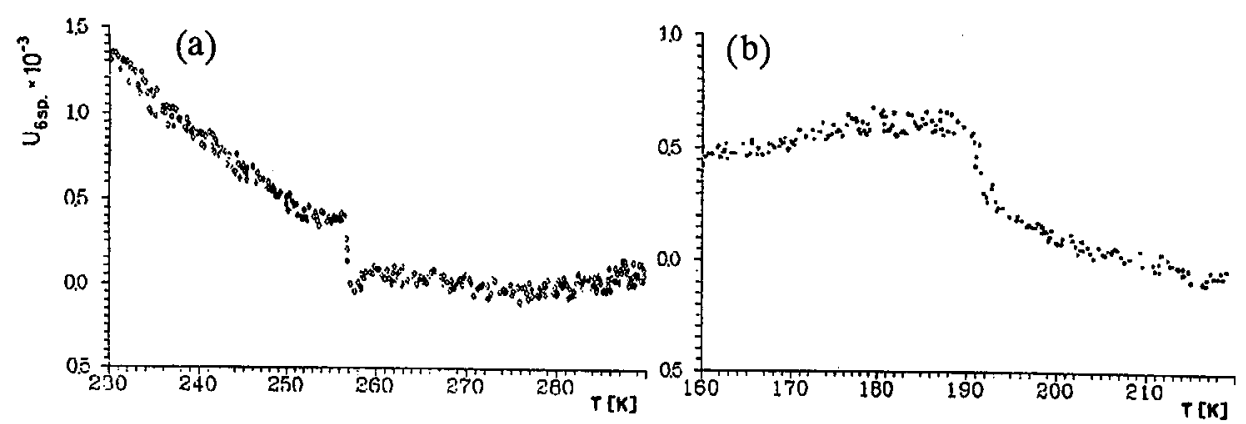

Fig. 5. Spontaneous deformation of (TEA) ${ }_{2} \mathrm{CuCl}_{4}$ crystal for [110] direction (a) in the region of structural phase transition, (b) near overcritical region.

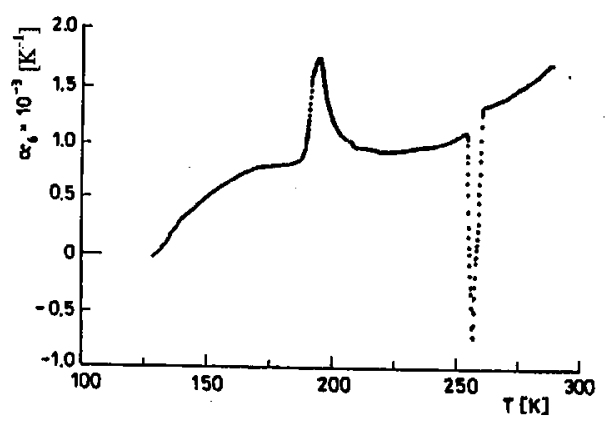

Fig. 6. Temperature dependence of (TEA) ${ }_{2} \mathrm{CuCl}_{4}$ crystal thermal expansion coefficient along the [110] direction.

continuous low-temperature anomaly which can be ascribed to the overcritical trace of the isostructural phase transition.

The relative jump of the crystal volume $\Delta V / V$ calculated on the basis of presented above dilatometric measurements at structural phase transition (at $258 \mathrm{~K}$ ) is equal to 0.0045 . This value is close to that obtained by Tylczynski and Biskupski [15] by dilatometric measurements in [100] and [111] directions $(\Delta V / V=0.00505)$.

\section{Phenomenological model}

In order to describe a phase transition sequence: a structural first-order phase transition from $P 4_{2} / n m c$ to $P n n a$ space group with doubling of the unit cell volume - an isostructural phase transition, the two-component order parameter can be taken into account in the free energy expansion. In these expansion there should be considered components containing the order parameter up to the tenth power and components describing the crystal interaction with external stress (we used expansion similar to that proposed by Levaniuk and Sannikov [20] for improper ferroelectrics)

$$
\begin{aligned}
\Phi= & \alpha_{1}\left(\eta^{2}+\xi^{2}\right)+\alpha_{2}\left(\eta^{2}+\xi^{2}\right)^{2}+\alpha_{3}(\eta \xi)^{2}+\alpha_{4}\left(\eta^{2}+\xi\right)^{3}+\alpha_{5}\left(\eta^{2}+\xi^{2}\right)^{4} \\
& +\alpha_{6}\left(\eta^{2}+\xi^{2}\right)^{5}-\delta_{1} \eta \xi \sigma-\rho \sigma^{2}
\end{aligned}
$$


where $\eta, \xi$ are the order parameter components, $\sigma$ denotes the tensor component of the mechanical stress, $\alpha_{1}, \alpha_{2}, \alpha_{3}, \alpha_{4}, \alpha_{5}, \alpha_{6}$ are the expansion coefficients (we assume that only $\alpha_{1}$ depends on temperature and $\alpha_{6}>0$ ), $\rho$ is the component of the elastic susceptibility and $\delta_{1}$ denotes coupling coefficients of deformations and order parameters. Expansion (2) is invariant with respect to symmetry elements of $\mathrm{P}_{2} / \mathrm{nmc}$ space group.

Conditions for the minimum of two-component order parameter expansion are the following:

$$
\left\{\begin{array} { l } 
{ \frac { \partial \Phi } { \partial \eta } = 0 , } \\
{ \frac { \partial \Phi } { \partial \xi } = 0 , }
\end{array} \quad ( 3 \mathrm { a } ) \quad \left\{\begin{array}{l}
\frac{\partial^{2} \Phi}{\partial \eta^{2}}>0, \\
\frac{\partial^{2} \Phi}{\partial \xi^{2}}>0,
\end{array} \quad(3 \mathrm{~b}) \quad\left|\begin{array}{cc}
\frac{\partial^{2} \Phi}{\partial \eta^{2}} & \frac{\partial \Phi}{\partial \eta} \\
\frac{\partial \Phi}{\partial \xi} & \frac{\partial^{2} \Phi}{\partial \xi^{2}}
\end{array}\right|>0\right.\right.
$$

Taking advantage of condition (3a) for the mechanically free crystal we have the following equations:

$$
\begin{aligned}
& \eta\left[\alpha_{1}+2 \alpha_{2}\left(\eta^{2}+\xi^{2}\right)+\alpha_{3} \xi^{2}+3 \alpha_{4}\left(\eta^{2}+\xi^{2}\right)^{2}+4 \alpha_{5}\left(\eta^{2}+\xi^{2}\right)^{3}\right. \\
& \left.\quad+5 \alpha_{6}\left(\eta^{2}+\xi^{2}\right)^{4}\right]=0 \\
& \xi\left[\alpha_{1}+2 \alpha_{2}\left(\eta^{2}+\xi^{2}\right)+\alpha_{3} \eta^{2}+3 \alpha_{4}\left(\eta^{2}+\xi^{2}\right)^{2}+4 \alpha_{5}\left(\eta^{2}+\xi^{2}\right)^{3}\right. \\
& \left.\quad+5 \alpha_{6}\left(\eta^{2}+\xi^{2}\right)^{4}\right]=0 .
\end{aligned}
$$

Equations (4) give solutions

$$
\begin{aligned}
& \eta=0 \text { and } \xi=0, \\
& \eta=0 \text { and } \xi \neq 0, \quad \alpha_{1}+\left(2 \alpha_{2}+\alpha_{3}\right) \xi^{2}+3 \alpha_{4} \xi^{4}+4 \alpha_{5} \xi^{6}+5 \alpha_{6} \xi^{8}=0 \\
& \xi=0 \text { and } \eta \neq 0, \quad \alpha_{1}+\left(2 \alpha_{2}+\alpha_{3}\right) \eta^{2}+3 \alpha_{4} \eta^{4}+4 \alpha_{5} \eta^{6}+5 \alpha_{6} \eta^{8}=0 \\
& \eta \neq 0, \xi \neq 0, \eta^{2}=\xi^{2} \\
& \quad \alpha_{1}+\left(4 \alpha_{2}+\alpha_{3}\right) \eta^{2}+12 \alpha_{4} \eta^{4}+32 \alpha_{5} \eta^{6}+80 \alpha_{6} \eta^{8}=0 .
\end{aligned}
$$

The first of the solutions (Eq. (5)) corresponds to a high-temperature phase with spontaneous deformation $u=0$, the other solutions correspond to the low-temperature phase. Solution (8) corresponds to the ferroelastic phases. If structural phase transition in (TEA) ${ }_{2} \mathrm{CuCl}_{4}$ crystals observed at $258 \mathrm{~K}$ is a transition to improper ferroelastic phase as suggested Mahoui and co-workers [11, 12], the solutions (8) would be taken into consideration.

The crystal deformation

$$
u=\delta_{1} \eta \xi+2 \rho \sigma \text {. }
$$

Putting Eq. (8) into Eq. (9) we have

$$
u= \pm \delta_{1} \eta^{2}+2 \rho \sigma \text {. }
$$

Equations (10) shows that the spontaneous deformation is proportional to square order parameter. 
Elastic susceptibility in a paraelastic phase

$$
\chi=2 \rho,
$$

whereas in a ferroelastic phase

$$
\chi=\frac{\delta_{1} \eta^{2}}{2\left[\alpha_{1}+\left(4 \alpha_{2}+\alpha_{3}\right) \eta^{2}+12 \eta^{4}+32 \eta^{6}+80 \eta^{8}\right]}+2 \rho .
$$

In order to determine the coordinates of phase transitions limits coordinates in six-dimensional space of $R\left(\alpha_{1}, \alpha_{2}, \alpha_{3}, \alpha_{4}, \alpha_{5}, \alpha_{6}\right)$, minimum conditions (3) of free energy expansion (2) were numerically analysed.

A phase diagram illustrating the results of numerical analysis of minimum condition of expansion (2) in the plane of $\left(\alpha_{1}, \alpha_{2}^{\prime}\right)$, where $\alpha_{2}^{\prime}=\frac{1}{2}\left(4 \alpha_{2}+\alpha_{3}\right)$ for $\alpha_{3}=1, \alpha_{4}=0.25, \alpha_{5}=-0.225$, and $\alpha_{6}=1 / 16$, is shown in Fig. 7. Lines 1 and 2 indicate the stability limit of paraelastic phase $0(\eta=\xi=0)$ and improper ferroelastic phase $1\left(\eta_{1}^{2}=\xi_{1}^{2} \neq 0\right)$, lines 3 and 4 represent the stability limits of improper ferroelastic phase 1 and improper ferroelastic phase $2\left(\eta_{2}^{2}=\xi_{2}^{2} \neq 0\right)$. The end point $K$ of the lines 3 and 4 is a critical point. Lines 5 and 6 indicate the stability limits of paraelastic phase 0 and improper ferroelastic phase 1 . The point where lines 1 and 2 meet is a tricritical point where the first-order structural phase transition converts into that of the second order.

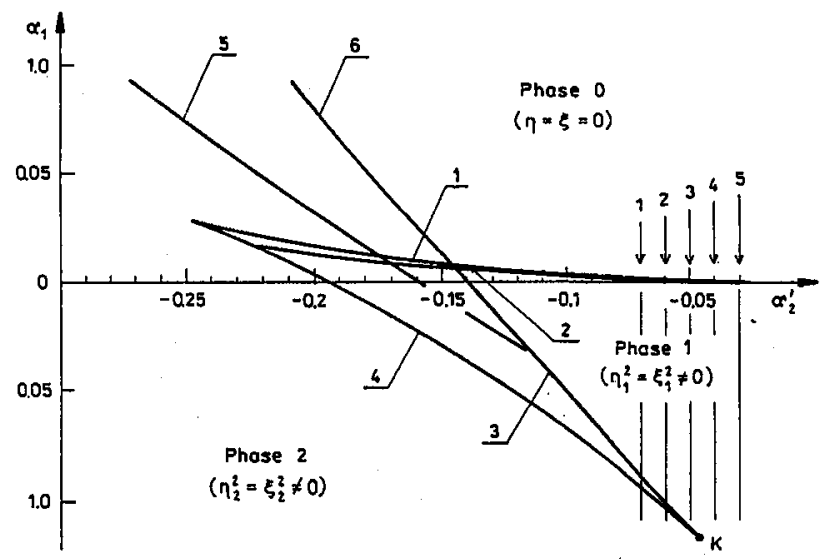

Fig. 7. Phase transition lines in $\left(\alpha_{1}, \alpha_{2}^{\prime}\right)$ plane, where $\alpha_{2}^{\prime}=\frac{1}{2}\left(4 \alpha_{2}+\alpha_{3}\right), \alpha_{3}=1$, $\alpha_{4}=0.25, \alpha_{5}=-0.225$, and $\alpha_{6}=1 / 16$ for the model described with free energy expansion (2). Stability limits of the phases are marked by the solid lines. Point $K$ denotes the end point of isostructural phase transition lines (critical point). Vertical lines denote cross-sections of the phase diagram for which dependencies presented in Figs. 8-10 were calculated.

An area on the right side of the critical $K$ point will be called the overcritical region. It is worth noting that there is an $\alpha_{2}^{\prime}$ range where the structural transition of the first order and the overcritical phenomena can be observed (cross-sections 4 and 5 in Fig. 7). We believe that physical properties of (TEA) ${ }_{2} \mathrm{CuCl}_{4}$ crystal correspond to this particular range. 


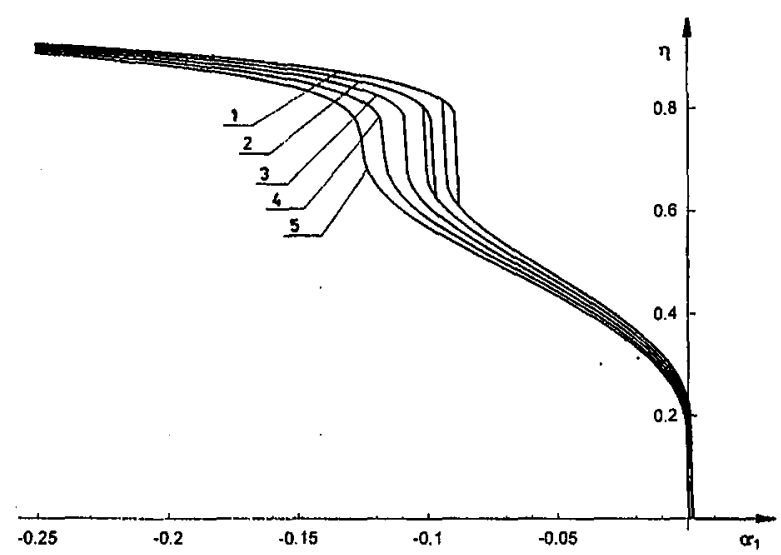

Fig. 8. Temperature dependence of the spontaneous deformation for improper ferroelastics resulting from the model described with free energy expansion (2) in the vicinity of the critical point: curves 1,2, and 3 were calculated for isostructural phase transition existence area, curves 4 and 5 for the overcritical region. The thermal hysteresis are marked.

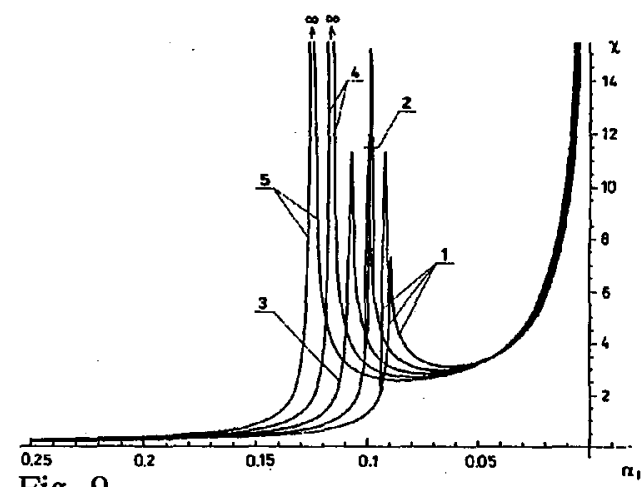

Fig. 9

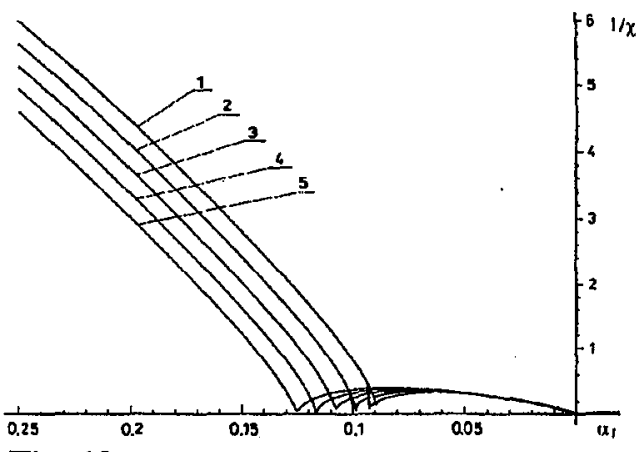

Fig. 10

Fig. 9. Temperature dependencies of the elastic susceptibility for improper ferroelastics resulting from the free energy expansion (2) in the vicinity of the overcritical point. The numbers denote cross-sections presented in Fig. 7. The thermal hysteresis are marked. Fig. 10. Temperature dependencies of the reverse elastic susceptibility for improper ferroelastics resulting from the free energy expansion (2) in the vicinity of the overcritical point. The numbers denote cross-sections presented in Fig. 7 and thermal hysteresis are marked.

Temperature dependence of the spontaneous deformation in the region of critical point (for $\alpha_{2}^{\prime}=-0.07,-0.06,-0.05,-0.04$, and -0.03 ) is shown in Fig. 8 . The jumps and temperature hysteresis of the crystal spontaneous deformation at structural and isostructural phase transitions can be seen on the curves 1,2 , and 3. Spontaneous deformation in the region on the right side of the critical point is represented in Fig. 8 by the curves 4 and 5. There is a jump of deformation at structural phase transition and continuous anomaly in the overcritical region. 
It is worth noting that spontaneous deformation anomalies observed in [001] directions are one order bigger than those observed in [110] directions. Similar conclusions can be drawn from dilatometric measurements in [100] and [111] directions [15].

In the overcritical region, with the temperature decreasing, the spontaneous deformation obtained from the analysis of potential (2) increases, whereas that determined from the experiment decreases. We think that the reason for this difference is a domain structure transformation in the overcritical region. In Eq. (10) there are two spontaneous deformations with opposite signs which correspond to opposite ferroelastic domains. This is interesting since a similar behaviour of the spontaneous polarisation [6] was observed in MAPCB crystals, a proper ferroelectric exhibiting overcritical phenomena.

Temperature dependence of the elastic susceptibility and reverse elastic susceptibility around the critical point calculated from the expansion (2) are shown in Figs. 9 and 10 respectively. In order to verify experimentally the relation, the sound velocity in the (TEA) ${ }_{2} \mathrm{CuCl}_{4}$ crystal should be measured. This can be performed either by a resonance method or Brillouin scattering measurements.

\section{Conclusion}

i) Low-temperature anomalies of physical properties of (TEA) ${ }_{2} \mathrm{CuCl}_{4}$ crystals can be treated as overcritical trace of isomorphous phase transition.

ii) Physical properties of (TEA) ${ }_{2} \mathrm{CuCl}_{4}$ crystals can be described by potential (2), but spontaneous deformation $u_{6}$ described by presented model is one order smaller than that observed in [001] direction.

iii) It is interesting to find an external factor to lead the crystal through the critical point.

iv) The order parameter fluctuations play a very important role in the overcritical region. The fluctuations should cause the anomaly of specific heat and the critical light and phonon scattering. This encourages us to continue studies on thermal conduction, elastic properties and light scattering in (TEA) ${ }_{2} \mathrm{CuCl}_{4}$ crystals and other materials with overcritical phenomena.

Ending, we would like point out that changing in potential (2) the term $\delta_{1} \eta \xi \sigma$ describing interactions of the order parameter with external stress by the term $\delta_{2} \eta \xi E$ describing interaction of the order parameter with external electric field, one can describe phase transitions to improper ferroelectric phase. In these case temperature dependence of spontaneous polarisation and electric susceptibility will be the same as presented above dependencies of spontaneous deformation and elastic susceptibility.

Adding the term $\delta_{2} \eta \xi E$ into potential (2) we can describe phase transition to improper ferroelastoelectric phase. In all cases mentioned above, the phase diagrams and temperature dependencies of entropy changes and specific heat for electrically and mechanically free crystals will have the same character as that calculated for improper ferroelastic [21]. 


\section{References}

[1] A. Jayaraman, Phys. Rev. 137, A179 (1965).

[2] M. Tatsumi, T. Matsuo, H. Saga, S. Seki, Bull. Chem. Soc. Jpn. 52, 728 (1979).

[3] K. Gesi, K. Ozawa, Phys. Lett. A 49, 283 (1974).

[4] M. Iwata, Y. Ishibasi, J. Phys. Soc. Jpn. 61, 4615 (1992).

[5] B.A. Strukov, R. Poprawski, S.A. Taraskin, J. Mróz, Phys. Status Solidi A 143, K.9 (1994).

[6] A. Liber, S. Matyjasik, R. Poprawski, Ferroelectrics 185, 91 (1996).

[7] B.A. Strukov, S.A. Taraskin, E.P. Regula, R. Poprawski, J. Mróz, J. Korean Phys. Soc. 32, 5216 (1998).

[8] T.P. Melia, R. Merrifield, J. Chem. Soc. (a), no. 7, 1166 (1970).

[9] T.P. Melia, R. Merrifield, J. Chem. Soc. (a), no. 9, 1258 (1971).

[10] A. Mahoui, J. Lapasset, J. Moret, P. Saint-Gregoire, Z. Kristallogr. 210, 125 (1995).

[11] A. Mahoui, J. Lapasset, J. Moret, P. Saint-Gregoire, Ferroelectrics 175, 79 (1996).

[12] A. Mahoui, J. Lapasset, D.G. Sannikov, J. Moret, P. Saint-Gregoire, Z. Phys. B 99, 543 (1996).

[13] T. Kawata, T. Aoyama, S. Ohba, Acta Crystallogr. C 49, 137 (1993).

[14] P. Saint-Gregoire, H.N. Borlando, A. Mahoi, J. Lapasset, J. Moret, Ferroelectrics 190, 57 (1997).

[15] Z. Tylczyński, P. Biskupski, J. Korean Phys. Soc. 32, 5235 (1998).

[16] Z. Tylczyński, P. Biskupski, Solid State Commun. 102, 385 (1997).

[17] Yu. Gufan, Strukturnye fazovye perekhody, Nauka, Moskva 1982 (in Russian).

[18] J.C. Toledano, P. Toledano, The Landau Theory of Phase Transitions, Word Sci., Singapore 1987.

[19] A. Liber, R. Poprawski, in: I Krajowa Konferencja: Metody i Systemy Komputerowe w Badaniach Naukowych i Projektowaniu Inżynierskim, Kraków 1997, Ed. T. Szmuc, Krakowskie Centrum Informatyki Stosowanej, Kraków 1997, p. 193 (in Polish).

[20] A.P. Levaniuk, D.G. Sannikov, Usp. Fiz. Nauk 112, 502 (1974) (in Russian).

[21] R. Poprawski, M. Drulis, A. Liber, Ferroelectric Lett., in press. 